\title{
Эпитаксиальный карбин: аналитические результаты
}

\author{
() С.Ю. Давыдов \\ Физико-технический институт им. А.Ф. Иофрфе Российской академии наук, \\ 194021 Санкт-Петербург, Россия \\ E-mail: Sergei_Davydov@mail.ru \\ Поступила в Редакцию 3 декабря 2018 г. \\ В окончательной редакции 28 февраля 2019 г. \\ Принята к публикации 28 февраля 2019 г.
}

Методом функций Грина в приближении сильной связи получены аналитические выражения для электронных спектров и плотностей состояний двух структурных модификаций карбина (кумулена и полиина). В качестве подложек рассмотрены металл и полупроводник, приведены соответствующие оценки перехода заряда. С учетом внутри- и межатомного кулоновского отталкивания получены критерии появления волны зарядовой плотности в свободном и эпитаксиальном карбине. Приведены аналитические выражения для фононного спектра свободного карбина. Полученные результаты сопоставлены с результатами численных расчетов других авторов.

Ключевые слова: карбин, кулоновское взаимодействие, металлическая и полупроводниковая подложки, переход заряда.

DOI: 10.21883/FTP.2019.07.47876.9036

\section{1. Введение}

Одномерные углеродные структуры, или карбины, исследуются экспериментально (см. обзор [1], а также [2-4]) и, значительно интенсивнее, теоретически (см. [1,3,5-14] и ссылки, приведенные там). Хотя первое упоминание о карбине относится к 1921 г., серьезное изучение его свойств началось после бурного старта графеновой тематики [1]. Различают два вида карбинов: металлический кумулен с двойными связями $(\ldots=\mathrm{C}=\mathrm{C}=\ldots)$ и полупроводниковый полиин с чередующимися одинарными и тройными связями $(\ldots \equiv \mathrm{C}-\mathrm{C} \equiv \mathrm{C}-\mathrm{C} \equiv \ldots)$ (рис. 1$)$. До недавнего времени синтезированы были лишь сравнительно короткие цепочки, например, полиин из 44 атомов углерода (см. ссылки в [3]). На свойства таких коротких цепочек кардинально влияют: число атомов в цепочках; закрепление их концов атомами водорода, какими-либо молекулярными группами или графеновыми нанолентами, необходимое для стабилизации цепочек (end-capped carbon chains); окружающая среда, что свидетельствует о возможности использовать карбины в качестве сенсо-

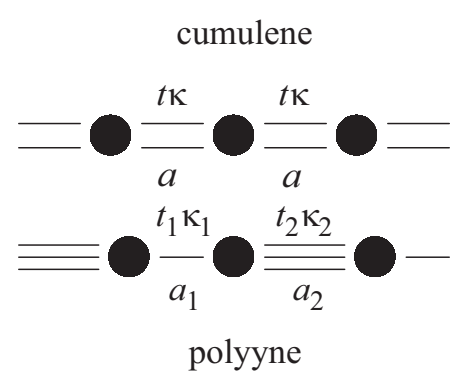

Рис. 1. Карбин: геометрия и межатомные связи в кумулене и полиине (схема). ров $[4,12]$. В настоящее время удалось создать сверхдлинную линейную цепочку из 6000 атомов, встроенную в двустенную углеродную нанотрубку (УНТ) [2,3]. Таким образом, задача о бесконечной углеродной цепочке утратила чисто академический характер.

Теоретически изучались электронные спектры [3,5-7,9-13], электронный транспорт [4,5,8,9], упругость и фононы $[3,7-10,13,14]$, оптические свойства [13], сверхпроводимость [11], магнетизм [7], переход Пайерлса между кумуленом и полиином [3,7,10,13]. Как правило, эти и другие работы по карбину представляют собой численные расчеты с использованием DFT (density functional theory) или квантово-химических методов. Все эти расчеты (за исключением работы $[14]^{1}$ ) относятся к свободному карбину. Здесь мы рассмотрим бесконечные цепочки кумулена и полиина, характеризуемые соответственно однородным и неоднородным распределениями $\pi$-электронов вдоль цепочки (ось $z$ ), как в свободном состоянии, так и адсорбированные на металле и полупроводнике. При этом используется теория сильной связи и метод функций Грина, что позволяет получить результаты в аналитическом виде. Основное внимание уделено электронным характеристикам свободного и эпитаксиального карбинов. Обсуждаются также упругие и фононные характеристики.

\section{2. Функции Грина эпикарбина}

Для описания эпитаксиального карбина (эпикарбина) используем адсорбционный подход [15], выстраивая це-

\footnotetext{
${ }^{1}$ В [14] рассматривалось влияние на рамановские спектры взаимодействия углеродной цепочки с окружающей ее УНТ. Там же имеются ссылки на более ранние работы, где рассмотрены, например, короткие углеродные цепочки в растворе, взаимодействие цепочек с островками Ag.
} 
почку не из атомов углерода, а из адатомов. Единичному (изолированному) адатому углерода сопоставляется функция Грина

$$
g(\omega)=(\omega-\varepsilon-\Lambda(\omega)+i \Gamma(\omega))^{-1}
$$

Здесь $\omega-$ энергетическая переменная, $\varepsilon-$ энергия $p_{x, y}$ состояний атома углерода (в дальнейшем полагаем $\varepsilon=0), \quad \Gamma(\omega)=\pi V^{2} \rho_{\text {sut }}(\omega)-$ функция полуширины квазиуровня адатома $\left(\rho_{\text {sut }}(\omega)-\right.$ плотность состояний подложки, $V-$ матричный элемент взаимодействия адатом-подложка), $\Lambda(\omega)=$ $=\pi^{-1} P \int_{-\infty}^{\infty} \Gamma\left(\omega^{\prime}\right) d \omega^{\prime} /\left(\omega-\omega^{\prime}\right)-$ функция сдвига квазиуровня адатома ( $P$ - символ главного значения). Исходя из уравнения Дайсона, которое в операторном виде для функции Грина эпитаксиального эпикарбина $G$ можно представить как $G=g+g T G$, где $T$ - оператор $\pi$-взаимодействия $p_{x, y}$-орбиталей ближайших соседей (б. с.), получим для кумулена

$$
\begin{gathered}
G_{\text {cum }}(\omega, k)=g(\omega) / D_{\text {cum }}(\omega, k), \\
D_{\text {cum }}(\omega, k)=1-2 g t \cos (k a),
\end{gathered}
$$

где $t$ - энергия перехода (собственное значение оператора $T), a=1.282 \AA$ - длина двойной связи $\mathrm{C}=\mathrm{C}$ [5], $k$ - волновой вектор для движения электрона вдоль оси $z$, зона Бриллюэна для кумулена определяется неравенством $\left|k_{\text {cum }}\right| \leq \pi / a$. По Харрисону [16] энергия перехода $t=\left|\eta_{p р л}\right|\left(\hbar^{2} / m_{0} a^{2}\right)$, где $\hbar-$ приведенная постоянная Планка, $m_{0}$ - масса свободного электрона, $\left|\eta_{\text {ррл }}\right|=0.63$, так что $t=2.92$ эВ.

$\mathrm{B}$ полиине одинарная связь $\mathrm{C}-\mathrm{C}$ имеет длину $a_{1}=1.265 \AA$, а тройной связи $\mathrm{C} \equiv \mathrm{C}$ отвечает длина $a_{2}=1.301 \AA$ [5]. Тогда соответствующие энергии перехода $t_{1,2}=\left|\eta_{p р л}\right|\left(\hbar^{2} / m_{0} a_{1,2}^{2}\right)$ равны $t_{1}=3.00$ эВ и $t_{2}=2.84$ эВ. Полагая $\Delta a=a_{1}-a_{2}$, $2 a=a_{1}+a_{2} \Delta t=t_{1}-t_{2}, 2 t^{\prime}=t_{1}+t_{2}$ и вводя безразмерные параметры $\alpha=\Delta a / 2 a$ и $\tau=\Delta t / 2 t^{\prime}$, из уравнения Дайсона найдем функцию Грина для полиина:

$$
\begin{gathered}
G_{\mathrm{pol}}(\omega, k)=g(\omega) / D_{\mathrm{pol}}(\omega, k), \\
D_{\mathrm{pol}}(\omega, k)=D_{1}(\omega, k)-i D_{2}(\omega, k),
\end{gathered}
$$

где

$$
\begin{gathered}
D_{1}(\omega, k)=1-2 g(\omega) t^{\prime} A_{1}(k), \\
D_{2}(\omega, k)=2 g(\omega) t^{\prime} A_{1}(k), \\
A_{1}(k)=\cos (\alpha k a) \cos (k a)-\tau \sin (\alpha k a) \sin (k a), \\
A_{2}(k)=\sin (\alpha k a) \cos (k a)+\tau \cos (\alpha k a) \sin (k a) .
\end{gathered}
$$

Зона Бриллюэна для полиина определяется неравенством $\left|k_{\text {pol }}\right| \leq \pi / 2 a$. Отметим, что с хорошей точностью можно положить $t^{\prime}=t$, что мы в дальнейшем и будем использовать в численных оценках.

\section{3. Свободный карбин}

Полагая в (1) $\Lambda(\omega)=0$ и $\Gamma(\omega)=0^{+}$, получим вместо (2) и (3) функции Грина $G_{\mathrm{cum}}^{0}(\omega, k)$ и $G_{\mathrm{pol}}^{0}(\omega, k)$. Электронные спектры кумулена и полиина есть

$$
\begin{gathered}
\omega_{\text {cum }}(k)=-2 t \cos (k a), \quad\left|k_{\text {cum }}\right| \leq \pi / a, \\
\omega_{\text {pol }}^{ \pm}(k)= \pm R(k), \quad R(k)=2 t^{\prime} \sqrt{A_{1}^{2}(k)+A_{2}^{2}(k)}, \\
\left|k_{\mathrm{pol}}\right| \leq \pi / 2 a .
\end{gathered}
$$

Ширина зоны проводимости кумулена $W_{\text {cum }}=4 t$ $\sim 12$ эВ, ширина проводящей и валентной зон полиина в линейном по малым параметрам $\alpha$ и $\tau$ приближении $W_{\mathrm{pol}} \approx 4 t^{\prime}(1-\tau) \sim 12$ эВ, что по порядку величины совпадают с результатами работ [5,9]. Отметим, что для $\sigma$-зон также справедливы формулы (7) и (8), но с заменой $t, t_{1,2}$ на $t^{*}=\left|\eta_{s p}\right|\left(\hbar^{2} / m_{0} a^{2}\right)$, $t_{1,2}^{*}=\left|\eta_{s p}\right|\left(\hbar^{2} / m_{0} a_{1,2}^{2}\right)$, где для $s p$-гибридизации вводится множитель $\left|\eta_{s p}\right|=3.19$ [16]. Тогда $t^{*} / t=t_{1,2}^{\prime \prime} / t_{1,2}$ $=\eta_{\text {ррл }} / \eta_{s p} \approx 5.06$.

Спроецировав $\omega_{\text {cum }}(k)$ на зону Бриллюэна полиина, получим закон дисперсии в виде $\omega_{\mathrm{cum}}^{ \pm}(k)= \pm 2 t \cos (k a)$. Вследствие малости отношений $\Delta a / a \approx-0.03$ и $\Delta t / 2 t \approx 0.03$, значимые различия спектров (7) и (8) наблюдаются только при $|k| \rightarrow \pi / 2 a .^{2}$ У полиина при $|k|=\pi / 2 a$ в электронном спектре имеется щель $\Delta_{\text {pol }}=2 R(\pi / 2 a)=2 \Delta t \approx 0.32$ эВ; у кумулена щель отсутствует. Полученное нами значение $\Delta_{\text {pol }}$ в точности совпадает с результатом работы [7] и близко к 0.37 эВ [13] и 0.407 эВ [9]. Отметим, однако, что в литературе наблюдается значительный разброс данных о щели для бесконечной цепочки: $\Delta_{\text {pol }} \sim 0.2-8.5$ эВ $[3,6,12]$. Так как $\Delta_{\mathrm{pol}} \approx 4 t|\Delta a| / a$, ширина щели зависит от деформации полиина (см. далее). Отметим, что для $\sigma$-зон $\Delta_{\text {pol }}^{\prime \prime}=\Delta_{\text {pol }}\left(\eta_{s p} / \eta_{\text {pрл }}\right) \approx 1.62$ эВ.

Интегрируя $G_{\text {cum }}^{0}(\omega, k)$ по зоне Бриллюэна кумулена, получим

$$
G_{\mathrm{cum}}^{0}(\omega)=\frac{1}{\sqrt{\omega^{2}-4 t^{2}}},
$$

так что плотность состояний свободного кумулена $\rho_{\text {cum }}^{0}=-\pi^{-1} \operatorname{Im} G_{\text {cum }}^{0}$ (в расчете на одну проекцию спина) имеет вид

$$
\rho_{\text {cum }}^{0}(\omega)= \begin{cases}\frac{1}{\pi \sqrt{4 t^{2}-\omega^{2}}}, & \omega^{2} \leq 4 t^{2} \\ 0, & \omega^{2}>4 t^{2}\end{cases}
$$

В собственном карбине уровень Ферми $\varepsilon_{\mathrm{F}}=0$, так что $\pi_{x}$ - и $\pi_{y}$-зоны полузаполнены. Действительно, при нулевой температуре $n_{\mathrm{cum}}^{0}=2 \int_{-2 t}^{0} \rho_{\text {cum }}^{0}(\omega) d \omega=1$.

\footnotetext{
2 Мы здесь не приводим графиков дисперсионных зависимостей для кумулена и полиина, так как первая из них относится к любой одноатомной цепочке, а вторая заметно отличается от первой лишь вблизи границы зоны Бриллюэна. Отметим, что численные расчеты $[5,7,9]$ дают качественно те же зависимости.
} 
Функцию Грина свободного полиина (3) можно представить в виде

$$
G_{\mathrm{pol}}^{0}(\omega, k)=\left(\omega-2 t A_{1}(k)-i 2 t A_{2}(k)\right)^{-1} .
$$

Из структуры функции Грина (11) очевидно, что плотность состояний $\rho_{\text {pol }}^{0}(\omega)$ нельзя получить в аналитическом виде. Поэтому, воспользовавшись сходством зонных спектров $\omega_{\text {cum }}^{ \pm}(k)$ и $\omega_{\text {pol }}^{ \pm}(k)$, мы предлагаем в качестве $\rho_{\mathrm{pol}}^{0}(\omega)$ использовать следующее выражение:

$$
\rho_{\mathrm{pol}}^{0}(\omega)= \begin{cases}\frac{C}{\pi \sqrt{4 t^{2}-\omega^{2}}}, & \Delta t^{2} \leq \omega^{2} \leq 4 t^{2} \\ 0, & \omega^{2}<\Delta t^{2}, \omega^{2}>4 t^{2}\end{cases}
$$

где нормировочный множитель $C=[1-(2 / \pi)$ $\times \arcsin (\Delta t / 2 t)]^{-1}$. Отметим подобие различий плотностей состояний кумулена и полиина, с одной стороны, и плотностей состояний графена и графеноподобного соединения (ГПС), с другой [17]: у кумулена и графена щели в спектре отсутствуют, тогда как у полиина и ГПС щели имеются. У полиина наличие щели определяется двумя типами связей, у ГПС - химическим различием атомов в элементарной ячейке.

\section{4. Эпикарбин на металле}

Будем считать, что плотность состояний металлической подложки $\rho_{m}(\omega)=$ const, так что $\Gamma(\omega)=\Gamma_{m}$ $=\pi V_{m}^{2} \rho_{m}=$ const, $\Lambda_{m}(\omega)=0$ и $g=\omega+i \Gamma_{m}\left(V_{m}-\right.$ матричный элемент взаимодействия карбин-металл) $[15,16]$. Тогда для кумулена на металле получаем

$$
\begin{aligned}
& G_{\mathrm{cum}}^{m}(\omega, k)=\left(\omega-2 t \cos (k a)+i \Gamma_{m}\right)^{-1}, \\
& \rho_{\mathrm{cum}}^{m}(\omega, k)=\frac{1}{\pi} \frac{\Gamma_{m}}{(\omega-2 t \cos (k a))^{2}+\Gamma_{m}^{2}} .
\end{aligned}
$$

В режиме слабой связи кумулена с подложкой $\left(t^{2} \gg \Gamma_{m}^{2}\right)$ аппроксимируем плотность состояний свободного кумулена (10) выражением $[\delta(\omega+2 t)+\delta(\omega-2 t)] / 2, \quad$ где $\delta(\ldots)-\delta$-функция Дирака. Плотность состояний адсорбированного на металле кумулена можно тогда записать как

$$
\begin{gathered}
\rho_{\text {cum }}^{m}(\omega) \approx \frac{1}{2}\left[\rho_{+}^{m}(\omega)+\rho_{-}^{m}(\omega)\right], \\
\rho_{ \pm}^{m}(\omega)=\frac{1}{\pi} \frac{\Gamma_{m}}{(\omega \pm 2 t)^{2}+\Gamma_{m}^{2}} .
\end{gathered}
$$

Функция $\rho_{\text {cum }}^{m}(\omega)$ приведена на рис. 2 в сопоставлении с функцией $\rho_{\text {cum }}^{0}(\omega)$. Из рис. 2 следует, что взаимодействие с металлической подложкой приводит к размытию плотности состояний свободного кумулена.

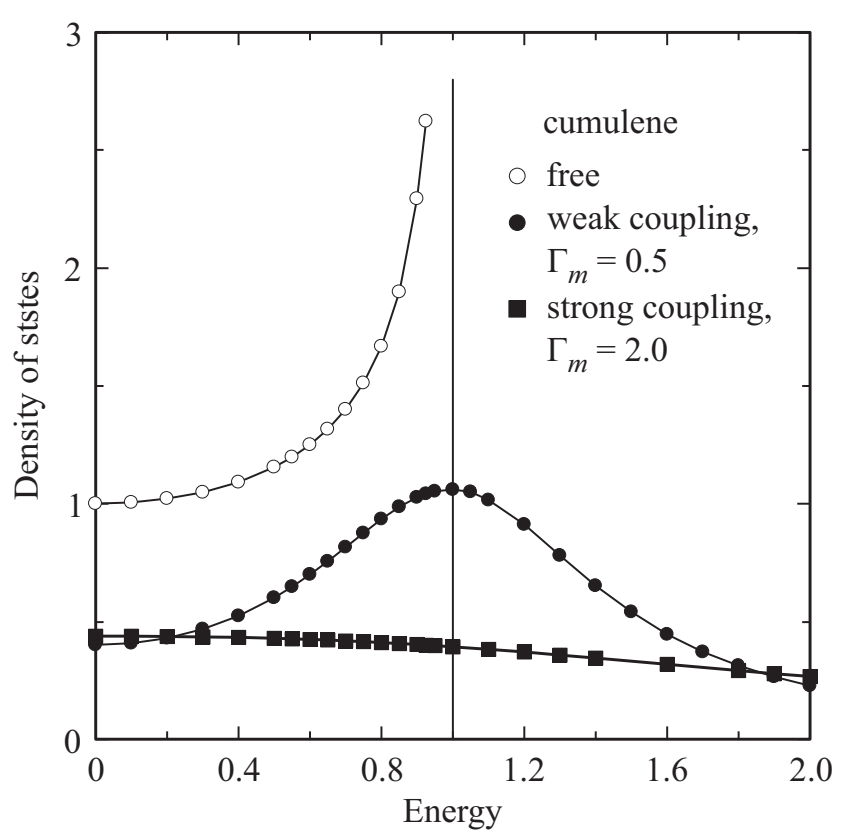

Рис. 2. Плотности состояний свободного $\rho_{\text {cum }}^{0}(\omega)$ (светлые кружки) и адсорбированного на металле $\rho_{\text {cum }}^{m}(\omega)$ кумулена в режимах слабой (темные кружки, $\left.\Gamma_{m}=0.5\right)$ и сильной (темные квадраты, $\Gamma_{m}=2$ ) связи с подложкой. Изображены только правые половины четных функций $\rho_{\text {cum }}^{0}(\omega)$ и $\rho_{\text {cum }}^{m}(\omega)$. Все энергетические величины приведены в ед. $2 t$, плотности состояний - в ед. $(2 t)^{-1}$.

Расходимости на границах зоны исчезают, а вместо них появляются максимумы, высота которых понижается с увеличением $\Gamma_{m}$ (на рис. 2 изображен только максимум для положительной энергии). Соответствующее (15) число заполнения адатома кумелена $n_{\text {cum }}^{m}$ при нулевой температуре равно

$$
n_{\mathrm{cum}}^{m} \approx \frac{1}{2}\left(n_{+}^{m}+n_{-}^{m}\right), \quad n_{ \pm}^{m}=\frac{2}{\pi} \operatorname{arccot} \frac{ \pm 2 t-\varepsilon_{\mathrm{F}}}{\Gamma_{m}} .
$$

Зависимость заряда $Z_{\text {cum }}^{m}=1-n_{\text {cum }}^{m}$ от положения уровня Ферми представлена на рис. 3. Там же для квазисвободного кумулена изображена функция $Z_{\text {cum }}^{0}\left(\varepsilon_{\mathrm{F}}\right)=1-n_{\text {cum }}^{0}\left(\varepsilon_{\mathrm{F}}\right)=(2 / \pi) \arcsin \left(-\varepsilon_{\mathrm{F}} / 2 t\right)$ при $\left|\varepsilon_{\mathrm{F}}\right| \leq 2 t \quad$ и $\quad Z_{\text {cum }}^{0}\left(\varepsilon_{\mathrm{F}}\right)=+1(-1) \quad$ соответственно при $\varepsilon_{\mathrm{F}}<-2 t\left(\varepsilon_{\mathrm{F}}>2 t\right)^{3}$. Из рис. 3 следует, что уже слабое взаимодействие с подложкой вызывает значительное

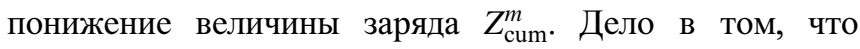
размытие плотности состояний кумулена (при $|\omega|>2 t$ имеем $\rho_{\text {cum }}^{m}(\omega) \neq 0$, а $\rho_{\text {cum }}^{0}(\omega)=0$ (рис. 2)) приводит к увеличению числа заполнения $n_{\text {cum }}^{m}$, следовательно, к уменьшению заряда $Z_{\text {cum }}^{m}$.

\footnotetext{
3 Здесь и далее под квазисвободным карбином (quasi-free-standing carbyne) мы будем понимать ситуацию, когда карбин взаимодействует с подложкой настолько слабо, что его плотность состояний не изменяется. При этом, однако, будем считать, что между карбином и подложкой разрешено туннелирование электронов, а положение уровня Ферми системы $\varepsilon_{\mathrm{F}}$ диктуется подложкой.
} 


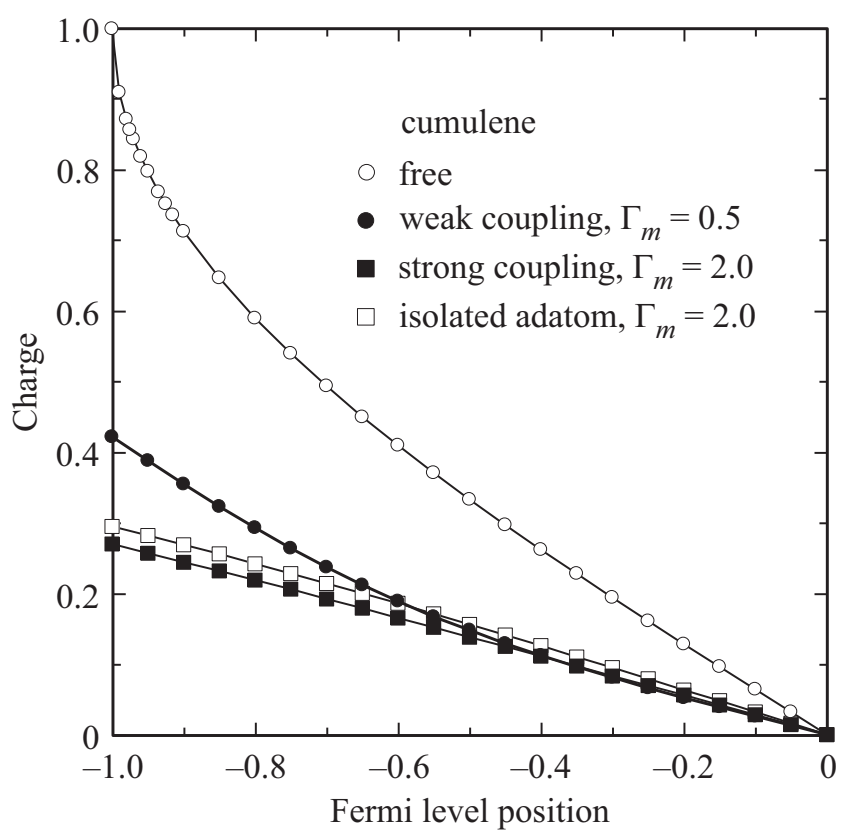

Рис. 3. Зависимости зарядов адатомов свободного кумулена $Z_{\text {cum }}^{0}$ (светлые кружки), адсорбированного на металле кумулена $Z_{\text {cum }}^{m}$ в режимах слабой (темные кружки, $\left.\Gamma_{m}=0.5\right)$ и сильной (темные квадраты, $\Gamma_{m}=2$ ) связи с подложкой и изолированного адатома углерода $Z_{a}^{m}$ (светлые квадраты, $\Gamma_{m}=2$ ) от положения уровня Ферми $\varepsilon_{\mathrm{F}}$. Функции $Z_{\mathrm{cum}}^{0}\left(\varepsilon_{\mathrm{F}}\right), Z_{\mathrm{cum}}^{m}\left(\varepsilon_{\mathrm{F}}\right)$ и $Z_{a}^{m}\left(\varepsilon_{\mathrm{F}}\right)$ являются нечетными; $Z_{\text {cum }}^{m}=1$ и $Z_{\text {cum }}^{0}=-1$ при $\varepsilon_{\mathrm{F}} \leq-1$ и $\varepsilon \geq 1$ соответственно. Все энергетические величины приведены в ед. $2 t$.

Переходя к режиму сильной связи кумулена с подложкой $\left(t^{2} \ll \Gamma_{m}^{2}\right)$, из (14) получим

$\rho_{\mathrm{cum}}^{m}(\omega) \approx \rho_{a}^{m}(\omega)\left(1+\frac{2 t^{2}}{\omega^{2}+\Gamma_{m}^{2}}\left(\frac{4 \omega^{2}}{\omega^{2}+\Gamma_{m}^{2}}-1\right)\right)$,

где $\rho_{a}^{m}(\omega)=\Gamma_{m} / \pi\left(\omega^{2}+\Gamma_{m}^{2}\right)$ - плотность состояний одиночного адатома углерода на металле. Сопоставление функций (15) и (17) (рис. 2) показывает, что с переходом в режим сильной связи максимумы $\rho_{\text {cum }}^{m}(\omega)$ практически исчезают. Соответствующее плотности состояний (17) число заполнения адатома кумулена $n_{\text {cum }}^{m}$ при нулевой температуре равно

$$
n_{\mathrm{cum}}^{m} \approx n_{a}^{m}-\frac{4 t^{2} \varepsilon_{\mathrm{F}} \Gamma_{m}}{\pi\left(\varepsilon_{\mathrm{F}}^{2}+\Gamma_{m}^{2}\right)^{2}}
$$

где $n_{a}^{m}=(2 / \pi) \operatorname{arccot}\left(-\varepsilon_{\mathrm{F}} / \Gamma_{m}\right)-$ число заполнения одиночного атома углерода, адсорбированного на металле [15]. Зависимости заряда $Z_{\text {cum }}^{m}$ от положения уровня Ферми $\varepsilon_{\mathrm{F}}$ изображены на рис. 3. Очевидно, что с ростом взаимодействия кумулен-подложка происходит уменьшение величины заряда. Это достаточно общий эффект, известный из теории адсорбции [15]: любое взаимодействие ведет к деполяризации адатома, т.е. к уменьшению величины его заряда. В этом можно убедиться, сопоставив заряды изолированного адатома углерода $Z_{a}^{m}$ и адатома кумулена $Z_{\text {cum }}^{m}$ (рис. 3 ).

Для числа заполнения атома квазисвободного полиина получим

$$
n_{\mathrm{pol}}^{m}=C \begin{cases}0, & \varepsilon_{\mathrm{F}}<-2 t, \\ 1+(2 / \pi) \arcsin \left(\varepsilon_{\mathrm{F}} / 2 t\right), & -2 t \leq \varepsilon_{\mathrm{F}} \leq-\Delta t, \\ 1, & -\Delta t<\varepsilon_{\mathrm{F}}<\Delta t, \\ 1+(2 / \pi) \arcsin \left(\varepsilon_{\mathrm{F}} / 2 t\right), & \Delta t \leq \varepsilon_{\mathrm{F}} \leq 2 t, \\ 2, & \varepsilon_{\mathrm{F}}>2 t .\end{cases}
$$

Из рис. 2 следует, что щель $\Delta_{\text {pol }} / 2 t \approx 0.06$ заметно проявляется только при $\varepsilon_{\mathrm{F}} / 2 t \approx 0.03$, где заряд $Z_{\text {pol }}^{m}$ обращается в нуль. Таким образом, в квазисвободном состоянии различием зависимостей $n_{\mathrm{pol}}^{m}\left(\varepsilon_{\mathrm{F}}\right)$ и $n_{\mathrm{cum}}^{m}\left(\varepsilon_{\mathrm{F}}\right)$, $Z_{\text {pol }}^{m}\left(\varepsilon_{\mathrm{F}}\right)$ и $Z_{\text {cum }}^{m}\left(\varepsilon_{\mathrm{F}}\right)$ можно пренебречь. Этот вывод тем более справедлив для случая сильной связи, когда металлическая подложка нивелирует все особенности плотностей состояний $\rho_{\mathrm{pol}}^{m}(\omega)$ и $\rho_{\mathrm{cum}}^{m}(\omega)$.

\section{5. Эпикарбин на полупроводнике}

В качестве $\rho_{\text {sut }}(\omega)$ для полупроводниковой подложки выберем модель, использованную в работе [18]:

$$
\rho_{s c}(\Omega)=B \begin{cases}\sqrt{-\Omega-E_{g} / 2}, & \Omega \leq-E_{g} / 2, \\ \sqrt{\Omega-E_{g} / 2}, & \Omega \geq E_{g} / 2, \\ 0, & |\Omega|<E_{g} / 2,\end{cases}
$$

где $\Omega=\omega-\varepsilon_{0}, \quad \varepsilon_{0}-$ энергия центра запрещенной зоны, ширина которой равна $E_{g}, B-$ коэффициент с размерностью эВ ${ }^{-3 / 2}$. Воспользовавшись выражением (20), получим функции полуширины $\Gamma_{s c}(\omega)=\pi V_{s c}^{2} \rho_{s c}(\omega)\left(V_{s c}\right.$ - матричный элемент взаимодействия карбин-полупроводник) и сдвига квазиуровня

$$
\Lambda_{s c}(\Omega)=B V_{s c}^{2} \begin{cases}F_{-}(\Omega), & \Omega<-E_{g} / 2, \\ F_{-}(\Omega)-F_{+}(\Omega), & |\Omega| \leq E_{g} / 2, \\ -F_{+}(\Omega), & \Omega>E_{g} / 2,\end{cases}
$$

где $F_{ \pm}(\Omega)=\pi \sqrt{ \pm \Omega+E_{g} / 2}$. По оценкам [18] для политипа $6 H-\mathrm{SiC}\left(E_{g}=3\right.$ эВ) коэффициент $B \approx 0.2$ эВ $^{-3 / 2}$, так что максимальное значение функции $\left|\Lambda_{s c}\right| \sim V_{s c}^{2}$ эВ.

Рассмотрим карбин, адсорбированный на полупроводнике, и начнем анализ со случая $\varepsilon_{0}=0$. Это наиболее интересный случай, так как при $\left|\varepsilon_{0}\right|>2 t+E_{g} / 2$ мы, с качественной точки зрения, возвращаемся к „металлической ситуации“, рассмотренной в предыдущем разделе. Так как величина $2 t\left(\sim 6\right.$ эВ) превосходит значения $E_{g} / 2$ даже для таких диэлектриков как $\mathrm{BN}\left(E_{g} \sim 6\right.$ эВ) и $\mathrm{SiO}_{2}$ $\left(E_{g} \sim 9\right.$ эВ), то центральная часть плотности состояний карбина перекрывается с запрещенной зоной подложки, 
а периферийные части плотности состояний - с валентной зоной и зоной проводимости. Так как $\Gamma_{s c}(\Omega)=0$ при $|\Omega| \leq E_{g} / 2$, плотность состояний кумулена в области запрещенной зоны подложки дается формулой (10), а плотность состояний полиина - формулой (12), где $\omega$ нужно заменить на $\bar{\Omega}=\Omega-\Lambda_{s c}(\Omega)$ и $4 t^{2}$ на $\left(E_{g} / 2\right)^{2}$. Таким образом, для числа заполнения адатома кумулена на полупроводнике имеем

$$
n_{\text {cum }}^{s c}=n_{\text {cum }}^{s c}(\text { v. b. })+n_{\text {cum }}^{s c}(\text { gap }),
$$

где $n_{\text {cum }}^{s c}(\mathrm{v} . \mathrm{b}$.$) - вклад валентной зоны подложки,$ $n_{\text {cum }}^{s c}$ (gap) - вклад запрещенной зоны. Воспользовавшись выражениями (10) и (14), легко получить

$$
\begin{gathered}
n_{\text {cum }}^{s c}(\text { v. b. })=\frac{a}{\pi^{2}} \int_{-\infty}^{\varepsilon_{\mathrm{F}}} d \omega \int_{-\pi / 2 a}^{\pi / 2 a} d k \\
\times \frac{\Gamma_{s c}(\omega)}{\left[\omega-2 t \cos (k a)-\Lambda_{s c}(\omega)\right]^{2}+\Gamma_{s c}^{2}(\omega)}, \\
n_{\text {cum }}^{s c}(\text { gap })=1+(2 / \pi) \arcsin \left(2 \varepsilon_{\mathrm{F}} / E_{g}\right) .
\end{gathered}
$$

Аналогичные выражения можно записать и для полиина. Из сравнения функций $\rho_{\text {cum }}^{0}(\omega)$ и $\rho_{\text {pol }}^{0}(\omega)$ ясно, однако, что различия зависимостей $n_{\text {cum }}^{s c}\left(\varepsilon_{\mathrm{F}}\right)$ и $n_{\mathrm{pol}}^{s c}\left(\varepsilon_{\mathrm{F}}\right)$ незначительны, так как $2 \Delta t / E_{g} \ll 1$ (во всяком случае, для широкозонных полупроводников) и $C-1 \approx \Delta t / \pi t \ll 1$. Следует, однако, подчеркнуть: если при $\varepsilon_{0} \neq 0$ щель $\Delta_{\text {pol }}$ полностью или хотя бы частично располагается внутри запрещенной зоны подложки, то полиин остается полупроводником. Если же щель перекрывается с зоной проводимости или валентной зоной („металлическая ситуация“), то полиин становится металлом. Если каким-либо образом (например, внешним электрическим полем) сдвигать щель из запрещенной зоны подложки в одну из разрешенных зон, можно осуществлять переход полупроводник-металл. Вообще говоря, рассматриваемая в этом разделе задача во многом аналогична задаче о гетеропереходе между ГПС и полупроводниковой подложкой, рассмотренной нами в работе [19].

\section{6. Учет кулоновского взаимодействия}

Вернемся к свободному карбину и рассмотрим роль внутриатомного $U$ и межатомного $G$ кулоновских взаимодействий электронов (расширенная модель Хаббарда), следуя работе [20]. В приближении Хартри-Фока эти взаимодействия можно записать в виде

$$
\begin{gathered}
U \sum_{i}\left(\hat{n}_{i \uparrow} n_{i \downarrow}+\hat{n}_{i \downarrow} n_{i \uparrow}-n_{i \uparrow} n_{i \downarrow}\right), \\
G \sum_{\langle i, j\rangle}\left(\hat{n}_{i} n_{j}+\hat{n}_{j} n_{i}-n_{i} n_{j}\right),
\end{gathered}
$$

где $\hat{n}_{i \sigma}-$ оператор числа заполнения $i$-го атома углерода в карбине, $n_{i \sigma}=\left\langle\hat{n}_{i \sigma}\right\rangle \quad(\sigma-$ спиновый индекс), $n_{i}=\sum_{\sigma} n_{i \sigma}=\left\langle\hat{n}_{i}\right\rangle,\langle\ldots\rangle-$ усреднение по основному состоянию, $\langle i, j\rangle$ означает суммирование по б.с. Если числа заполнения $n_{i}$ всех атомов карбина одинаковы и равны $n$, то в немагнитном случае $\left(n_{i \sigma}=n_{i-\sigma}=n / 2\right)$ энергия атомного уровня есть $\varepsilon+U n / 2+2 G n$. Если же числа заполнения соседних атомов 1 и 2 равны $n_{1}$ и $n_{2}$, то их уровни имеют энергии $\varepsilon_{1}=\varepsilon+U n_{1} / 2+2 G n_{2}$ и $\varepsilon_{2}=\varepsilon+U n_{2} / 2+2 G n_{1}$. Положим $n_{1}+n_{2}=2 n, n_{1}-n_{2}=2 v$ и учтем, что для свободного карбина $n=1$ и $\varepsilon^{*}=\varepsilon+U / 2+2 G=0$. Если $U, G \gg 1$, то энергия карбина (на один атом) в нулевом по $t$ приближении равна $E_{0}=(U / 2-2 G) v^{2}$. Отсюда следует, что при $G>U / 4$ энергетически выгодно состояние с $v \neq 0$ и $n_{1,2}=1 \pm v$, отвечающее волне зарядовой плотности (ВЗП). Легко показать, что с учетом $t$-взаимодействия спектры свободных кумулена и полиина имеют вид

$$
\begin{gathered}
\omega_{\mathrm{cum}}^{ \pm}(k)= \pm \sqrt{(U / 2-2 G)^{2} v^{2}+4 t^{2} \cos ^{2}(k a)}, \\
\omega_{\mathrm{pol}}^{ \pm}(k)= \pm \sqrt{(U / 2-2 G)^{2} v^{2}+A_{1}^{2}(k)+A_{2}^{2}(k)}, \\
\left|k_{\mathrm{pol}}\right| \leq \pi / 2 a .
\end{gathered}
$$

Отметим, что для графена в отсутствие экранировки $U=17.0$ эВ и $G=8.5$ эВ, с учетом экранировки $U=9.3$ эВ и $G=5.5$ эВ $[21]$.

Рассмотрим квазисвободный кумулен на металле $\left(t^{2} \gg \Gamma_{m}^{2}\right)$, положив $\varepsilon^{*}=\varepsilon_{\mathrm{F}}=0$, так что $n^{0}=1$ и $\left(Z_{\text {cum }}^{0}\right)_{1}=-\left(Z_{\text {cum }}^{0}\right)_{2}=v$. Тогда получаем уравнение вида

$$
2 t \sin (\pi v / 2)=(2 G-U / 2) v
$$

Необходимым условием существования решения $v \neq 0$ является выполнение неравенства $2 t<2 G-U / 2<\pi t$. Верхний предел продиктован областью энергий, занимаемой плотностью состояний кумулена (12), нижний предел отвечает существованию ВЗП. Если для оценки приписать вычисленные для графена значения $U$ и $G$ кумулену, то без учета экранировки получаем (в ед. эВ) достаточно сильное неравенство $5.84<8.50$, свидетельствующее о возникновении ВЗП. С учетом экранировки имеем слабое неравенство $5.84<6.35$, не позволяющее с уверенностью говорить о переходе однородного по заряду кумулена в ВЗП-состояние.

Переходя к режиму слабой связи (15) и (16), получаем уравнение

$$
\begin{aligned}
v=\frac{1}{\pi} & \left(\arctan \frac{(U / 2-2 G) v-2 t}{\Gamma_{m}}\right. \\
& \left.+\arctan \frac{(U / 2-2 G) v+2 t}{\Gamma_{m}}\right) .
\end{aligned}
$$

При $0<v \ll 4 t^{2}(2 G-U / 2)^{2}$ решение $v \neq 0$ существует при выполнении неравенства $2 G-U / 2>2 \pi t^{2} / \Gamma_{m}$. В режиме сильной связи $\left(t^{2} \ll \Gamma_{m}^{2}\right)$, отбрасывая для простоты 
второе (малое по сравнению с первым - см. рис. 2) слагаемое в (18), получим уравнение

$$
\Gamma_{m} \tan (\pi v / 2)=(2 G-U / 2) v
$$

так что решение $v \neq 0$ существует при $2 G-U / 2>\pi \Gamma_{m} / 2$. Вообще говоря, в теории адсорбции полуширину адатома $\Gamma_{m}$ предпочитают рассматривать как подгоночный параметр [15], хотя можно дать и теоретическую оценку (см., например, [17]). Воспользовавшись теми же значениями $U$ и $G$, что и выше, можно сказать, что при $\Gamma_{m}<4$ эВ переход в ВЗП-состояние возможен. Это неравенство заведомо выполняется для ван-дер-ваальсового взаимодействия кумулина с подложкой, для которого $\Gamma_{m} \leq 1$ эВ [22]. Подчеркнем, однако, что величина $\Gamma_{m}$ зависит не столько от „физики“, сколько от технологии формирования углеродной нити на поверхности подложки.

Если для квазисвободного полиина положить $C=1$, т.е. пренебречь отношением $\Delta t / \pi t \ll 1$, получим для условия существования ВЗП (при $\varepsilon^{*}=\varepsilon_{\mathrm{F}}=0, n^{0}=1$ ) уравнение (27). Так как, в соответствии с выводами разд. 4, при усилении связи с металлической подложкой качественные различия между кумуленом и полиином практически исчезают, критерии (28) и (29) можно применять и для полиина. Нетрудно показать [16], что во всех рассмотренных случаях переход в ВЗП-состояние ведет к выигрышу энергии системы $\delta E \sim-2 G v^{2}$.

\section{7. Упругость и фононный спектр карбина}

Рассмотрим свободный кумулен. В соответствии с теорией Харрисона [15,23] матричный элемент связи $s p$-орбиталей (ковалентная энергия) $V_{2}=\left|\eta_{s p}\right|\left(\hbar^{2} / m_{0} a^{2}\right)$, где $\left|\eta_{s p}\right|=3.19$, так что $V_{2}=14.79$ эВ. Энергия двухэлектронной связи кумулена в пренебрежении энергией металлизации равна $\left|E_{b}\right|=2 V_{2} / 3$, т. е. 9.86 эВ, что близко к значениям энергии связи 7.17 эВ [13] и энергии когезии $E_{\mathrm{cum}}^{\mathrm{coh}}=8.19$ эВ [7] (отметим, что $E_{\mathrm{pol}}^{\mathrm{coh}}>E_{\mathrm{cum}}^{\mathrm{coh}}$ на 2 мэВ). Константа центрального взаимодействия (без учета металлизации) равна $\kappa=4 V_{2} / a^{2}=36$ эВ $/ \AA^{2}$. При деформации $\varepsilon^{\prime}=\delta a / a=0.1$ получаем силу натяжения (на единицу длины) $F=\kappa \delta a=4.68$ эВ $/ \AA$, что близко к значению $F \sim 6$ эВ/A [7]. Отметим, что для графена (также без учета металличности) имеем $V_{2}=12.32$ эВ, $\left|E_{b}\right|=8.21$ эВ, $\kappa=24.44$ эВ/ $\AA^{2}$ [23]. Введенная в [13] жесткость растяжения $C \approx 94.67$ эВ/А связана с полученной нами константой $\kappa$ соотношением $C=\kappa a$, которое дает в 2 раза меньшее значение $C \approx 46.15$ эВ/A.

Для полиина получим $V_{21}=V_{2}\left(a_{1}\right)=15.19$ эВ, $V_{22}=$ $=V_{2}\left(a_{2}\right)=14.36$ э $\mathrm{B}, \kappa_{1}=\kappa\left(a_{1}\right)=4 V_{21} / a_{1}^{2}=37.97$ э $\mathrm{B} / \AA$, $\kappa_{2}=\kappa\left(a_{2}\right)=4 V_{22} / a_{2}^{2}=33.94$ эВ/A. Из постоянства силы натяжения $F$ вдоль цепочки имеем $\delta a_{1} \kappa_{1}=\delta a_{2} \kappa_{2}$, где $\delta a_{1(2)}$ - удлинение связи 1 (2). Отсюда получаем $\delta a_{2} / \delta a_{1}=\left(a_{2} / a_{1}\right)^{4}=1.12$.
Оценим величину деформационного изменения разницы длин тройной и одинарной связей, или BLA (bond length alternation). Так как BLA $=\tilde{a}_{2}-\tilde{a}_{1}$ (здесь и далее тильда обозначает деформированное состояние), получим BLA $\approx\left(a_{2}-a_{1}\right)+\left(a_{2} / a_{1}\right)^{4} a \tilde{\varepsilon}$, где $\tilde{\varepsilon}=\delta a / a-$ деформация. При $\tilde{\varepsilon}=0.1$ второе слагаемое $\mathrm{BLA} \approx 0.24 \AA$, что отлично согласуется с результатами расчетов $[9,10]$. Так как $\partial t_{1,2} / \partial a_{1,2}=-2 t_{1,2} / a_{1,2}$, для реакции щели полиина на деформацию получим $\delta \Delta_{\text {pol }} \approx 4\left[\left(a_{2} / a_{1}\right)^{4} t_{2}-t_{1}\right] a \tilde{\varepsilon}$. При $\tilde{\varepsilon}=0.1$ получаем $\delta \Delta_{\text {pol }} \sim 0.1$ эВ. Этот результат более чем на порядок выше, чем результаты расчетов [13], но настолько же ниже значений, полученных в $[7,8]$. Причины такого расхождения неясны. Подчеркнем, что для полиина на полупроводнике с помощью деформации можно в принципе (так же как и с помощью внешнего поля, разд. 5) смещать края щели относительно краев запрещенной зоны, осуществляя переход металл-изолятор.

Перейдем теперь к фононному спектру свободного карбина. Легко показать, что для продольных колебаний дисперсии частот для кумулена и полиина даются выражениями

$$
\begin{gathered}
M \bar{\omega}_{\mathrm{cum}}^{2}(q)=4 \kappa \sin ^{2}(q a / 2), \quad|q| \leq \pi / a, \\
M \bar{\omega}_{\mathrm{pol}}^{2}(q)=\left(\kappa_{1}+\kappa_{2}\right)\left(1 \pm \sqrt{1-\frac{4 \kappa_{1} \kappa_{2} \sin ^{2}(q a)}{\left(\kappa_{1}+\kappa_{2}\right)^{2}}}\right) \\
|q| \leq \pi / 2 a
\end{gathered}
$$

где $q$ - волновой вектор фонона, $M-$ масса атома углерода, знак плюс (минус) в (31) относится к оптической $\bar{\omega}_{\mathrm{pol}}^{\mathrm{OL}}$ (акустической $\left.\bar{\omega}_{\mathrm{pol}}^{\mathrm{AL}}(g)\right)$ продольной моде полиина. Отметим, что выражение (30) является стандартным законом дисперсии одноатомной цепочки. a (31) типично для двухатомной цепочки, где, однако, чередуются не массы атомов, а силовые константы [24]. Поэтому графиков зависимостей (30) и (31) мы здесь не приводим.

Из (30) следует, что частота продольной акустической моды кумулена на границе зоны Бриллюэна $\left|q_{\text {cum }}\right|=\pi / a$ равна $\bar{\omega}_{\text {cum }}^{\mathrm{LA}}(\pi / a)=2 \sqrt{\kappa / M} \approx 1800 \mathrm{~cm}^{-1}$, что вполне удовлетворительно согласуется с рассчитанным в [5] значением $\sim 2000 \mathrm{~cm}^{-1}$. Так как $\kappa_{1}+\kappa_{2} \approx 2 \kappa$, из (31) получаем, что частота продольной оптической моды полиина в центре зоны Бриллюэна $\bar{\omega}_{\text {pol }}^{\mathrm{LO}}(0)=\bar{\omega}_{\text {cum }}^{\mathrm{LA}}(\pi / a) \approx 1800 \mathrm{~cm}^{-1}$. Это значение отлично совпадает с частотой рамановского пика $1850 \mathrm{~cm}^{-1}$ [3], в [13] приводится значение $2084 \mathrm{~cm}^{-1}$, близкий результат приведен и в [7]. На границе зоны Бриллюэна получаем $\bar{\omega}_{\text {pol }}^{\mathrm{LO}}(\pi / 2 a) \approx 1320 \mathrm{~cm}^{-1}$ и $\bar{\omega}_{\text {pol }}^{\mathrm{LA}}(\pi / 2 a) \approx 1220 \mathrm{~cm}^{-1}$. Эти значения частот существенно отличаются от результатов работ $[7,12]$, где $\bar{\omega}_{\text {pol }}^{\mathrm{LO}}(\pi / 2 a) \sim 2000 \mathrm{~cm}^{-1}$ и $\bar{\omega}_{\text {pol }}^{\mathrm{LA}}(\pi / 2 a) \sim 1600 \mathrm{~cm}^{-1}[7,12]$. Более того, по данным $[7,12]$, дисперсия частоты $\bar{\omega}_{\text {pol }}^{\mathrm{LO}}(q)$ носит немонотонный характер с максимумом, расположенным внутри зоны Бриллюэна, тогда как зависимость (31) монотонна. 
Отметим, что все оценки этого раздела относятся к свободному карбину. Что же касается эпикарбина, то, не стремясь на данном этапе дать какие-либо конкретные оценки, сошлемся на работу [25], где показано, что для эпиграфена учет связи с подложкой вызывает увеличение центральной силовой константы $\kappa$, что, в частности, приводит к сдвигу $G$-пика в сторону более высоких частот. Можно ли этот вывод экстраполировать на эпикарбин, в настоящее время не ясно, так как по данным работ $[14,26]$ для цепочки полиина, встроенной в двустенную УНТ, сдвиг $G$-пика имеет противоположный знак.

\section{8. Заключение}

Итак, в работе получены аналитические выражения для электронных спектров и плотностей состояний кумулена и полиина, находящихся как в свободном, так и адсорбированных на металле и полупроводнике состояниях. С учетом внутри- и межатомного кулоновского отталкивания определены условия возникновения ВЗП. Для эпикарбина приведены оценки перехода заряда, для свободного карбина получен фононный спектр. В целом ряде случаев сопоставление полученных нами результатов с результатами расчетов других авторов показало вполне удовлетворительное согласие. Есть, однако, и серьезные расхождения (силовая константа кумулена, граничные фононные частоты полиина).

Отметим, что, помимо изучения собственно карбина, интерес исследователей все чаще привлекают более сложные (композитные) структуры: различные комбинации углеродных цепочек $[5,11,27]$; карбин, соединяющий графеновые наноленты $[4,8,9]$; карбин, встроенный в УНТ [3,14]. При этом, естественно, встает вопрос об учете взаимодействия карбина с контактирующими объектами. Поэтому рассмотренная здесь задача об эпикарбине представляется вполне своевременной. Более того, полученные нами простые аналитические выражения могут служить исходным материалом для рассмотрения композитных структур.

\section{Список литературы}

[1] F. Banhart. J. Nanotechnol., 6, 559 (2015).

[2] L. Shi, P. Rohringer, K. Suenaga, Y. Niimi, J. otakoski, J.C. Meyer, H. Peterlik, M. Wanko, S. Cahangirov, A. Rubio, Z.J. Lapin, L. Novotny, P. Ayala, T. Pichler. Nature Materials, 15, 634 (2016).

[3] L. Shi, P. Rohringer, M. Wanko, A. Rubio, S. Waserroth, S. Reich, S. Cambre, W. Wenseleers, P. Ayala, T. Pichler. Phys. Rev. Mater., 1, 075601 (2017).

[4] Z. Salman, A. Nair, S. Tung. Proc. 12th IEEE Intern. Conf. on Nano/Micro Engineered and Molecular Systems, April 9-12, 2017 (Los Angeles, USA).

[5] S. Tongay, R.T. Senger, S. Dag, S. Ciracil. Phys. Rev. Lett., 93, 136404 (2004).

[6] S. Yang, M. Kertesz. J. Phys. Chem. A, 110, 9711 (2006).
[7] S. Cahangirov, M. Topsakal, S. Ciraci. Phys. Rev. B, 82, 195444 (2010).

[8] B. Akdim, R. Pachter. ACS Nano, 5, 1769 (2011).

[9] O. Cretu, A.R. Botello-Mendez, I.M. Janowska, C. Pham-Huu, J.-C. Charlier, F. Banhart. Nano Lett., 13, 3487 (2013).

[10] V.I. Artyukhov, M. Liu, B.I. Yakobson. Nano Lett., 14, 4224 (2014).

[11] C.H. Wong, R. Lortz, E.A. Buntov, R.E. Kasimova, A.F. Zatsepin. Sci. Rep., 7 (1), 15815 (2017).

[12] E. Mostaani, B. Monserrat, N.D. Drummond, C.J. Lambert. Phys. Chem. Chem. Phys., 18, 14810 (2016).

[13] J. Li, S. Meng, H. Lu, T. Tohyama. arXiv: 1808.00794.

[14] M. Wanko, S. Cahangirov, L. Shi, P. Rohringer, Z.J. Lapin, L. Novotny, P. Ayala, T. Pichler, A. Rubio. Phys. Rev. B, 94, 195422 (2016).

[15] С.Ю. Давыдов. Теория адсорбции: метод модельных гамильтонианов (СПб., Изд-во СПбГЭТУ „ЛЭТИ“ 2013).

[16] W.A. Harrison. Phys. Rev. B, 27, 3592 (1983).

[17] С.Ю. Давыдов. ФТТ, 58, 779 (2016).

[18] С.Ю. Давыдов. ФТП, 48, 49 (2014).

[19] С.Ю. Давыдов. ФТТ, 58, 1182 (2016).

[20] С.Ю. Давыдов. ФТТ, 59, 1650 (2017).

[21] T.O. Wehling, E. Saswoglu, C. Friedrich, A.I. Lichtenstein, M.I. Katsnelson, S. Blügel. Phys. Rev. Lett., 106, 236805 (2011).

[22] С.Ю. Давыдов. ФТП, 52, 240 (2018).

[23] С.Ю. Давыдов, О.В. Посредник. ФТТ, 57, 819 (2015).

[24] Дж. Займан. Принцииы теории твердого тела (М., Мир, 1974).

[25] С.Ю. Давыдов. ФТТ, 59, 610 (2017).

[26] L.G. Moura, L.M. Malard, M.A. Carneiro, P. Venezuela, R.B. Capaz, D. Nishide, Y. Achiba, H. Shinohara, M.A. Pimenta. Phys. Rev. B, 80, 161401 (R) (2009).

[27] M. Liu, V.I. Artyukhov, H. Lee, F. Xu, B.I. Yakobson. ACS Nano, 7, 10075 (2013).

Редактор Г.А. Оганесян

\section{Epitaxial carbyne: analytical results}

\section{S.Yu. Davydov}

loffe Institute, 194021 St. Petersburg, Russia

Abstract Green's function method and tight-binding approximation are used to obtain analytical expressions for electronic spectra and corresponding densities of states for both carbyne structural modifications (cumulene and polyyne). Metal and semiconductor substrates are considered and the charge transfer estimates are fulfilled. Taking into account intra- and interatomic Coulomb repulsion criterions for the arising of charge density wave in free and epitaxial states of carbyne are proposed. Analytical expressions for free carbyne phonon spectra are found. All the obtaining results are compared with the numerical results of other authors. 